International Journal of Modern Physics B, Vol. 5, No. 20 (1991) 3289

(C) World Scientific Publishing Company

\title{
ERRATA
}

\section{BETHE APPROXIMATION SCHEME FOR ITINERANT ELECTRON SYSTEMS}

\author{
M. BECHI, R. LIVI and A. MONTORSI \\ [INT. J. MOD. PHYS. B, Vol. 5, No. 18 (1991) 2973-2987]
}

Page 2975: The first 2 lines of the second paragraph should read:

As a final remark, let us observe that an adaptation of the Bethe-Peierls method to itinerant fermion systems has already been proposed by Fazekas in Ref. 13.

Page 2975: Equation (4) should read:

$$
H_{\Lambda}=\sum_{\mathbf{i} \in \Lambda}\left(U D_{\mathbf{i}}-\mu\right) N_{\mathbf{i}}-\mu \sum_{\mathbf{i}} D_{\mathbf{i}}+t \sum_{\langle\mathbf{i}, \mathbf{j}\rangle \in \Lambda} A_{\mathbf{i}}^{\dagger} A_{\mathbf{j}}
$$

Page 2981: Equation (20) should read:

$$
\begin{aligned}
Z_{i}= & \exp \left(\beta q_{1} t \bar{\theta} \theta\right)\left[\left(1+\exp (\beta \mu) \exp \left(-\beta \frac{\left(q_{1} t\right)^{2}}{\mu} \bar{\theta} \theta\right)\right.\right. \\
& \left.+\exp (\beta \mu)(1+\exp \beta(\mu-U)) \exp \left(-\beta \frac{\left(q_{1} t\right)^{2}}{\mu-U} \bar{\theta} \theta\right)\right]
\end{aligned}
$$

